\title{
Photochemical model of Photodynamic Therapy applied to skin diseases by a topical photosensitizer
}

\author{
F. Fanjul-Vélez ${ }^{* a}$, I. Salas-García ${ }^{\mathrm{a}}$, L. A. Fernández-Fernández ${ }^{\mathrm{b}}$, M. López-Escobar ${ }^{\mathrm{c}}$, L. Buelta- \\ Carrillo $^{\mathrm{d}}$, N. Ortega-Quijano ${ }^{\mathrm{a}}$, J. L. Arce-Diego* ${ }^{\mathrm{a}}$ \\ ${ }^{a}$ Applied Optical Techniques Group, TEISA Department, University of Cantabria, Av. de los Castros \\ $\mathrm{s} / \mathrm{n}, 39005$ Santander (Spain) \\ ${ }^{b}$ Mathematics, Statistics and Computation Departament, University of Cantabria, Av. de los Castros \\ $\mathrm{s} / \mathrm{n}, 39005$ Santander (Spain) \\ ${ }^{c}$ Dermatology Department, Marqués de Valdecilla University Hospital, Av. Valdecilla s/n, 39008 \\ Santander, (Spain) \\ ${ }^{\mathrm{d}}$ Medical and Surgery Sciences Department, University of Cantabria, Av. Cardenal Herrera Oria s/n, \\ 39011 Santander (Spain)
}

\begin{abstract}
Photodynamic Therapy (PDT) provides a non-invasive, efficient and safe treatment for skin diseases with good cosmetic results. These characteristics make this technique more advantageous than radiotherapy or chemotherapy, which present limitations in a big number of lesions, are painful in many cases and produce non-satisfactory cosmetic results. We present the clinical results obtained at present by this optical technique and a photochemical model of the PDT process applied to the skin by means of a topical photosensitizer, in order to find the optimal PDT parameters. Optical propagation inside the tissue is calculated by means of the three dimensional Beer-Lambert law, due to its facility to be integrated in the differential equations system used to model the photochemical processes involved. With this information it is possible to obtain an initial estimation about the optimal drug dose and the optical power required.
\end{abstract}

Keywords: Photodynamic therapy, topical photosensitizer, skin disorders, singlet oxygen.

\section{INTRODUCTION}

Photodynamic Therapy (PDT) is successfully used in medical praxis to treat skin diseases like basocellular carcinoma or actinic keratoses. This technique is mainly based on photochemical reactions. The development of photoquimiotherapy involves the interaction between light, a photosensitizer and oxygen in ground state in order to provoke malignant tissue destruction. Photosensitizers are drugs which are administered by means of intravenous, oral or topical methods. Afterwards, the photosensitizer in ground state concentrates in cancer cells and can become active when light of a certain wavelength is directed onto the treated area. During the photochemical reaction, the photosensitizer in triplet state interacts with oxygen in ground state. This oxygen makes a transition to singlet state, which is the principal agent of cellular cytotoxicity [1].

The present application of PDT in medical praxis requires fixed protocols that depend on the photosensitizer supplier [2]. These protocols establish the optical parameters of the optical source, mainly power and exposition time, in order to assure that the treatment will be carried out with no collateral damage. In this case, every lesion receives the same kind of treatment. However, this fact avoids the use of the optimal parameters for the particular lesion we are dealing with.

In this work we use a photochemical model for the PDT process applied to skin diseases by means of Metvix@ topical photosensitizer. The different molecular reactions are modelled by means of a differential equations system. This system is solved via a numerical method, taking into account its stiff nature. The evolution of the PDT process can be predicted for different optical source parameters, and so the treatment effect could be known a priori.

\footnotetext{
*fanjulf@unican.es; irene.salas@alumnos.unican.es ; luisal.fdez@unican.es ; uc10909@humv.es; bueltal@unican.es ; ortegan@unican.es; arcedj@unican.es; phone+34 9422015 45; fax +34 9422018 73; www.teisa.unican.es/toa

Therapeutic Laser Applications and Laser-Tissue Interactions IV, edited by Ronald Sroka, Lothar D. Lilge,

Proc. of SPIE-OSA Biomedical Optics, SPIE Vol. 7373, 73730S - ( 2009 SPIE-OSA

CCC code: $1605-7422 / 09 / \$ 18 \cdot$ doi: $10.1117 / 12.831938$
} 
In section 2, the main skin pathologies and the clinical PDT application are described. In section 3, the results of our clinical PDT study are presented and discussed. The theoretical model for the PDT process and the results obtained are included in section 4 and 5, respectively. Finally, the general conclusions are shown in section 6.

\section{SKIN PATHOLOGIES AND CLINICAL APPLICATION OF PDT}

Nonmelanoma skin cancer is one of the most frequent malignant neoplasia in humans [3], and includes skin disorders such as basocellular carcinoma, squamous cell carcinoma or actinic keratoses. These pathologies are developed as a consequence of an accumulated solar exposition, so people suffering from them use to be more than 60 and with light skin. That is the reason why the most usual areas affected are the exposed body parts like the face, the scalp or the neckline.

Nonmelanoma skin cancer appears in the basal epidermal cells and it is originated without presenting a previous lesion. Metastasis risk is extremely low, but this kind of cancer is able to provoke great local tissue destruction. Squamous cell carcinoma can be basal or spinous, presents a fast growing process and it is more likely to invade other tissues. Previous lesions that degenerate in squamous cell carcinoma are actinic keratoses and Bowen disease [4]. Actinic keratoses, shown in Fig. 1, is the most frequent premalignant lesion and the main lesion that acts as a precursor of squamous cell carcinoma.

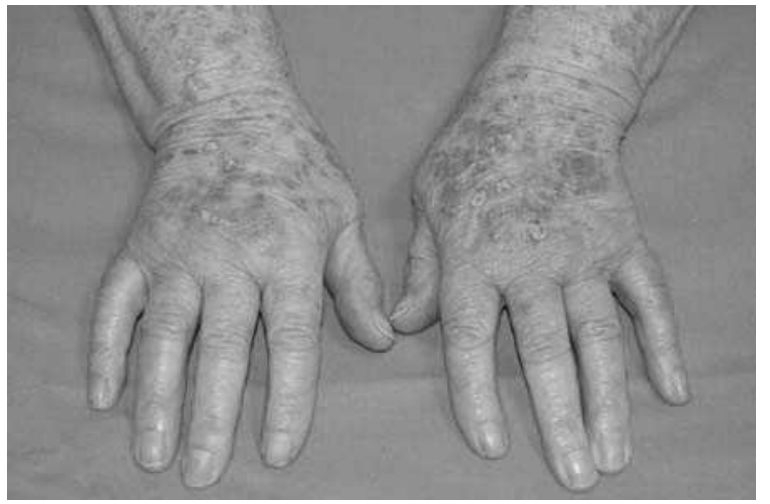

Fig. 1. Photo which shows the appearance of the lesions suffered as a consequence of actinic keratoses in a patient's hands.

There are several techniques of treatment for skin cancer like excision surgery, electrocoagulation, cryosurgery, micrographic Mohs surgery, radiotherapy, $\mathrm{CO}_{2}$ laser or pharmacological treatments like Imiquimod or 5-fluoracil. The choice of one of these techniques is motivated by the localization and size of the lesions, the previous treatments employed, the age and preferences of the patient, the skills of the practitioner and the rate cost-effectiveness. However, they present limitations in a big number of lesions. They are painful in many cases and the cosmetic results are in general non-satisfactory. In this way, PDT provides a non-invasive, efficient, safe and good cosmetic treatment.

Photodynamic Therapy (PDT) is mainly based on photochemical reactions. The development of photoquimiotherapy involves the interaction between light, a photosensitizer and oxygen in ground state in order to provoke malignant tissue destruction. Photosensitizers are drugs which are administered by means of intravenous, oral or topical methods. After some time, the photosensitizer in ground state concentrates in cancer cells and can become active when light of a certain wavelength is directed onto the treated area. During the photochemical reaction, the photosensitizer in triplet state interacts with oxygen in ground state. This oxygen makes a transition to singlet state, which is the principal agent of cellular cytotoxicity [1].

Our PDT research work will focus on the problems that this optical treatment technique currently reports in the case of the skin disorders described. The knowledge of these clinical deficiencies sets up the modelling approach requirements. In order to know and improve the clinical PDT procedure, we have studied different PDT treatment aspects together with the Department of Dermatology of the Marqués de Valdecilla University Hospital. Next section shows some of the most relevant results we have obtained. 


\section{CLINICAL STUDY OF PDT}

The PDT procedure involves the application of the topical photosensitizer (methyl aminolevulinate, Metvix $\odot$ ) followed by the exposure to its activating light source during eight or nine minutes. Then, the photochemical reactions will take place to selectively destroy tumour cells.

From April 2006 until January 2009, a total of 57 patients suffering from 71 skin disorders were treated at the PDT Service of the Marqués de Valdecilla University Hospital. The kind and number of skin diseases which have been treated are listed in Table 1, and as it is shown, the majority of patients suffered from actinic keratoses and basocellular carcinoma.

Table1. Distribution of skin diseases treated by PDT.

\begin{tabular}{|c|c|c|}
\hline \multicolumn{2}{|c|}{ Skin disorder } & Number of patients \\
\hline \multicolumn{2}{|c|}{ Actinic keratoses } & 37 \\
\hline \multirow{2}{*}{ Basocellular carcinoma } & Nodular & 23 \\
\cline { 2 - 3 } & Superficial & 10 \\
\hline \multicolumn{2}{|c|}{ Bowen disease } & 1 \\
\hline \multicolumn{2}{|c|}{ Total } & 71 \\
\hline
\end{tabular}

Regarding the response of the patients to the treatment, quite good results have been obtained without relevant side effects. These effects are mostly heat and slight pain ( $72 \%$ of the cases), and they appear in the great majority of the cases $(74 \%)$, as depicted in Fig.2. This feature constitutes, together with the non-invasive characteristic of PDT, the most important advantage opposite other tumour treatment techniques.
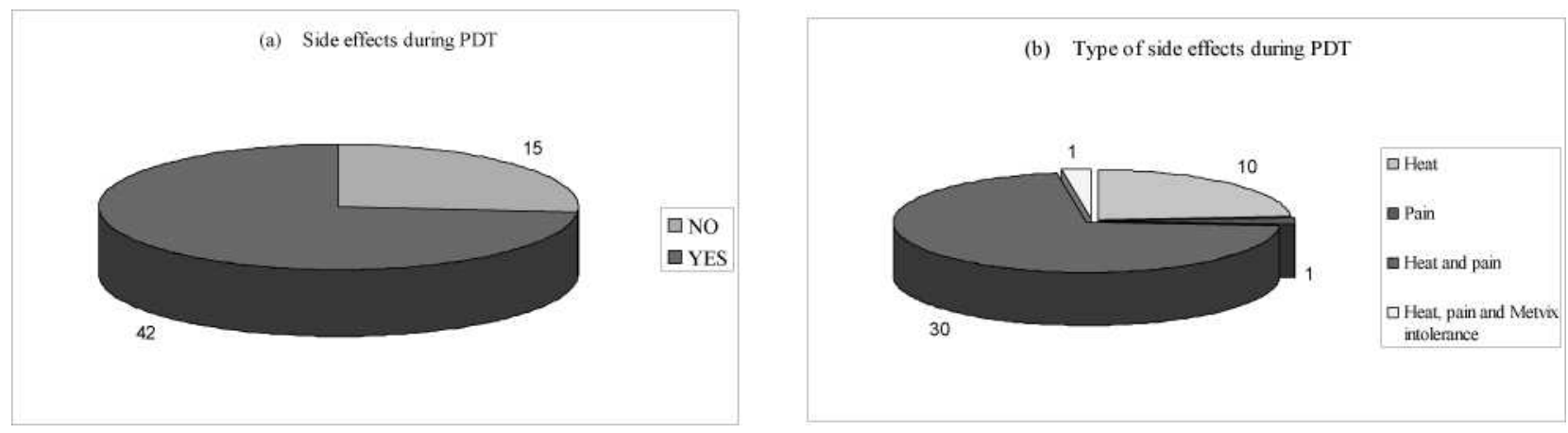

Fig.2. Side effects distribution during PDT, showing the number of patients who suffered side effects (a) and the type (b). 74 $\%$ of the whole treated patients (57) suffered side effects during PDT, mostly heat and slight pain which represent the $72 \%$ of whole registered cases.

Nonetheless, in several cases these results are not as successful as expected and the disorder appears again, as it is expressed by the recurrence index for the 3/6/12 months checkups that can be observed in Table 2 . The disease recurrence is the principal problem which must be solved, and more specifically in the case of nodular basocellular carcinoma, which represents the 60 per cent of the total recurrent cases (see Table 3).

Table 2. Recurrence index for the different checkups.

\begin{tabular}{|c|c|}
\hline Checkup date & Recurrence index (\%) \\
\hline 3 months & $3.125 \%$ \\
\hline 6 months & $5.55 \%$ \\
\hline 12 months & $0 \%$ \\
\hline
\end{tabular}


Table 3. Percentage of the total recurrence for each kind of skin disease.

\begin{tabular}{|c|c|}
\hline Skin disease & Percentage of the total recurrence cases \\
\hline Nodular basocellular carcinoma & $60 \%$ \\
\hline Superficial basocellular carcinoma & $20 \%$ \\
\hline Actinic keratoses & $20 \%$ \\
\hline
\end{tabular}

\section{THEORETICAL MODEL}

Modeling the PDT photochemical process requires the adequate knowledge of optical distribution inside the tissue. Among the several optical propagation models that exist, in this work we use the three dimensional Beer-Lambert law, due to its facility to be integrated in the photochemical system. The expression of this Beer-Lambert law is:

$$
I=I_{0} e^{-\alpha z} e^{-s\left(x^{2}+y^{2}\right)}
$$

The steady-state light distribution can then be calculated. The irradiance changes through the tissue sample and this is one of the parameters we should use in the photochemical model. For that purpose, it is necessary to know the photon density by means of the following equation:

$$
\rho=\frac{I(x, y, z)}{h \cdot f \cdot v}
$$

Prior predictive analysis was based on a simplified model for the photochemical processes involved. In this work, the photochemical model is based on a differential equations system that was already used by Foster et al [1] for a Type II PDT process in the time domain, and later improved by a research group of East Carolina and Maryland Universities that introduced the photobleaching of the photosensitizer by means of the term which includes the photobleaching constant, $\mathrm{k}_{\mathrm{pb}}$, in the equations bellow [5]. In this model, a set of rate equations are solved to describe molecular reactions that takes into account the transitions between states of the particles involved, like the photosensitizer or the oxygen. These equations are based on the Jablonski diagram for the singlet oxygen production, which is considered to be responsible for the malignant cells damage.

$$
\begin{gathered}
\frac{d\left[S_{0}\right]}{d t}=-v \rho \sigma_{p s a}\left[S_{0}\right]-k_{p b}\left[{ }^{1} O_{2}\right]\left[S_{0}\right]+\frac{\eta_{10}}{\tau_{1}}\left[S_{1}\right]+\frac{\eta_{30}}{\tau_{3}}[T]+\frac{\alpha s}{\tau_{3}}[T]\left[{ }^{3} O_{2}\right] \\
\frac{d\left[S_{1}\right]}{d t}=-\frac{1}{\tau_{1}}\left[S_{1}\right]+v \rho \sigma_{p s a}\left[S_{0}\right] \\
\frac{d[T]}{d t}=-\frac{\eta_{30}}{\tau_{3}}[T]-\frac{\alpha s}{\tau_{3}}[T]\left[{ }^{3} O_{2}\right]+\frac{\eta_{13}}{\tau_{1}}\left[S_{1}\right] \\
\frac{d\left[^{3} O_{2}\right]}{d t}=-\frac{\alpha s}{\tau_{3}}[T]\left[{ }^{3} O_{2}\right]+\frac{\eta_{0}}{\tau_{0}}\left[{ }^{1} O_{2}\right]+P \\
\frac{d\left[{ }^{1} O_{2}\right]}{d t}=-k_{p b}\left[S_{0}\right]\left[{ }^{1} O_{2}\right]-k_{c x}[R]\left[{ }^{1} O_{2}\right]-k_{s c}[C] i\left[{ }^{1} O_{2}\right]-\frac{\eta_{0}}{\tau_{0}}\left[{ }^{1} O_{2}\right]+\frac{\alpha s}{\tau_{3}}[T]\left[{ }^{3} O_{2}\right] \\
\frac{d[R]}{d t}=-k_{c x}\left[{ }^{1} O_{2}\right][R]+U
\end{gathered}
$$


In these equations, $\left[S_{1}\right]_{i}$ is the initial concentration of the photosensitizer in singlet excited state; $[T]_{i}$ is the initial concentration of photosensitizer in triplet excited state; $\left[{ }^{3} \mathrm{O}_{2}\right]_{i}$ the initial concentration of oxygen in ground state; $\left[{ }^{1} O_{2}\right]_{i}$ is the initial concentration of singlet oxygen; $[R]_{i}$ the initial concentration of singlet oxygen receptors; $[C]_{i}$ is the scavenger concentration; $\tau_{1}$ is the relaxation time from state $S_{1}$ to $S_{0} ; \tau_{3}$ is the relaxation time from state $T$ to $S_{0} ; \tau_{0}$ the relaxation time from state ${ }^{1} O_{2}$ to ${ }^{3} O_{2} ; \eta_{10}$ is the quantum yield of the transition from state $S_{1}$ to $S_{0} ; \eta_{13}$ is the quantum yield of the transition $S_{1}$ to $T ; \eta_{30}$ is the quantum yield of $T$ transition to $S_{0} ; \eta_{0}$ is the quantum yield of ${ }^{1} O_{2}$ transition to ${ }^{3} \mathrm{O}_{2} ; \alpha_{S}$ is the efficiency factor for energy transfer from $T$ to ${ }^{3} O_{2} ; k_{p b}$ stands for the biomolecular photobleaching rate; $k_{C x}$ is the biomolecular cytotoxicity rate; $v$ is light speed in tissue; $\rho$ is the photon density; $\sigma_{p s a}$ is the absorption cross-section of $S_{0}$ molecules; $P$ is the rate of oxygen diffusion and perfusion; and $U$ is the cell damage repair rate.

Throughout the photochemical process, complex reactions take place that involve multiple parameters, therefore predictions will be determined by this parameters choice. In this case, the values used have been obtained from several works [6].

\section{RESULTS}

The complete photochemical model was implemented for the particular case of PDT applied to the skin. The results obtained represent a graphical description of the different concentrations of photosensitizer, oxygen and singlet oxygen receptors for every point and temporal instant in the tissue sample. The parameters have been reported in several studies [7], [8], [9], [10].

By solving equations 3 to 8 for different values of photosensitizer concentration and photon density at every point of the tissue sample, we obtained the time dependence of the concentrations of photosensitizer, oxygen molecules and singlet oxygen receptors. We present one of the results obtained in Figure 3.

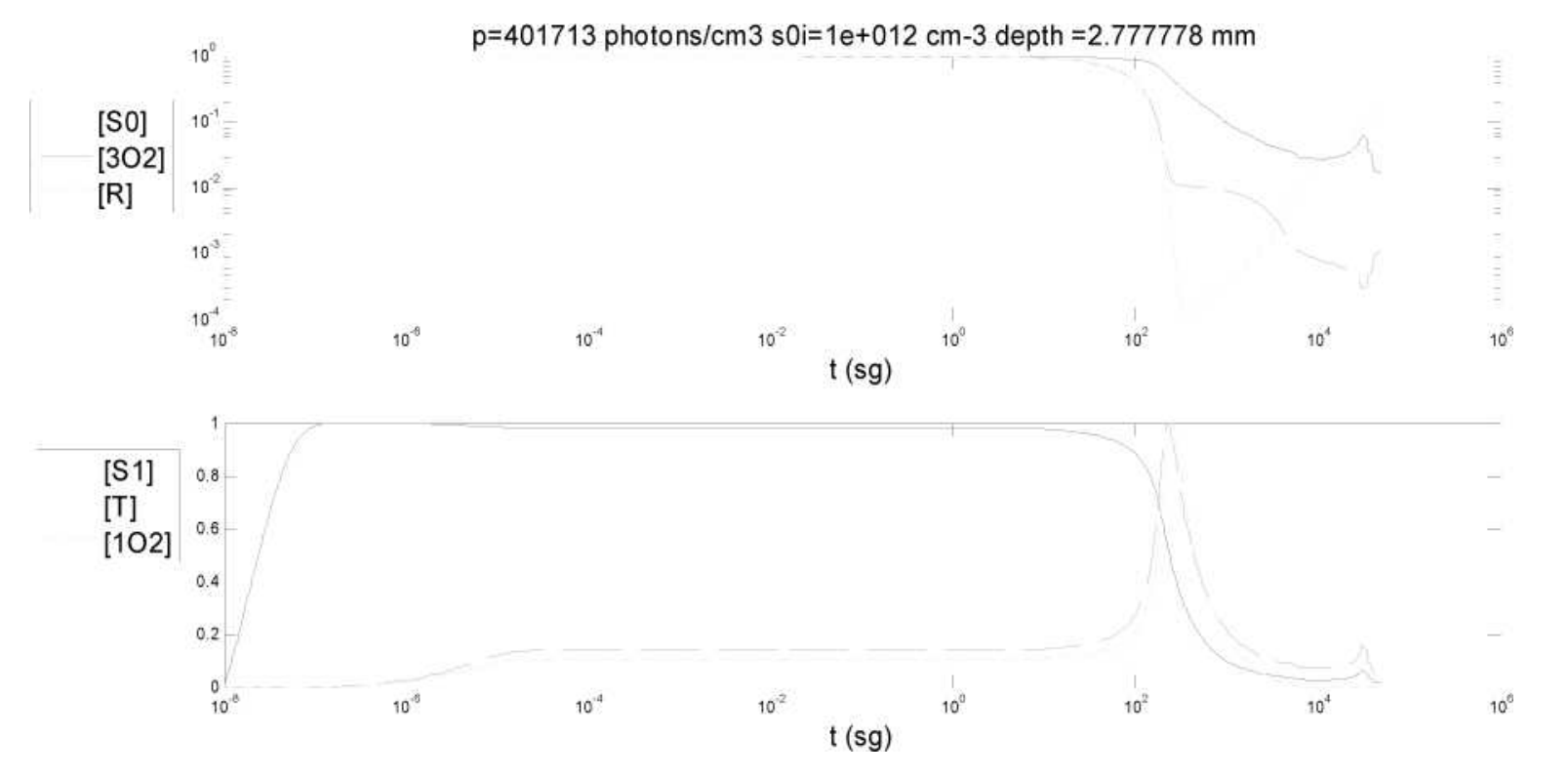

Fig. 3 This graphic describes the time dependence of the concentrations of photosensitizer in ground and excited state, of oxygen in ground and singlet state, and of singlet oxygen receptors for $I o=0.05 \mathrm{~W} / \mathrm{cm}^{2}$ and $\left[S_{0}\right]_{i}=1 \cdot 10^{12} \mathrm{~cm}^{-3}$ at 2.7 $\mathrm{mm}$ from tissue surface. 
As it can be observed in Fig. 3, the concentration of ground state molecules of photosensitizer, oxygen in ground state and receptors vary slowly in response to the activation light while the concentration of the triplet state molecules of photosensitizer and singlet oxygen begin to increase slowly until they reach a maximum value. It is supposed that this maximum in singlet oxygen concentration produces the desired cytotoxic effect, so we have made several simulations in order to adjust the optimal drug and light dose which will produce this effect in the shortest period of irradiation and for the largest depth of action in cancerous tissues.

The temporal dependence of the different concentrations involved in the photochemical reaction is shown in Fig. 3, and allow us to obtain an estimation about cytotoxicity and photobleaching by means of intracellular unoxidised receptors concentration and ground state molecules of photosensitizer, which could be used later in the development of cellular damage models.

The simulations executed permit to observe how the temporal instant for the maximum singlet oxygen varies depending on the drug dose and the optical power we use. Besides, we can know how much time is needed for different depths in the tissue. Therefore, as long as the light passes through the tissue, the irradiance is reduced as long as the intracellular oxidised receptors diminish, so the desired cellular damage would not take place in deeper depths in the tissue.

If we use an insufficient light or drug dose, the singlet oxygen concentration would not increase enough to produce the desired cytotoxic effect and tumour cells would not be damaged. As we increase both parameters, singlet oxygen concentration would increase and intracellular unoxidised receptors would decrease more rapidly. However, there are some limits related to the light dose choice, because it can provoke a dangerous thermal effect if it is too high. In our simulations we can observe that the light dose which allows the treatment to reach the maximum depth in the tissue with the desired cytotoxic effect is about $0.05 \mathrm{~W} / \mathrm{cm}^{2}$ using a photosensitizer concentration of $\left[S_{0}\right]_{i}=1 \cdot 10^{12} \mathrm{~cm}^{-3}$. These first results must be interpreted carefully, due to the great amount of parameters that are involved in these complex photoreactions. Subsequently future research works are required to improve the results obtained and to continue developing new accurate models.

\section{CONCLUSION}

Nonmelanoma skin cancer is one of the most frequent malignant neoplasia in humans, which includes basocellular carcinoma, squamous cell carcinoma or actinic keratoses. These skin disorders have grown during the last years due to an increase of solar exposition of the population, the increase of the longevity or the lack of ozone in the atmosphere.

Conventional techniques against these pathologies like surgery, electrocoagulation, cryosurgery, radiotherapy or even pharmacological solutions present some limitations that are successfully solved by PDT. Among PDT advantages over conventional treatment techniques, we could mention non-invasivity, selectivity and the lack of severe collateral effects.

Nowadays the process of PDT application is carried out by means of stiff protocols, and in this way the efficiency of the treatment for the particular problem is not assured, as we can observe in the clinical PDT study that we have presented. This clinical study identifies the recurrence of the skin disorders as the main inconvenient of this technique, especially in the case of nodular basocellular carcinoma. In order to remove this problem, it will be necessary to adjust all the parameters which are involved in the photochemical process like the photosensitizer dose or the optical power, so the cytotoxic effect could take place in the treated zone. Therefore, we use a photochemical model of the PDT process as a first approximation to develop an accurate model to be applied to preclinical planning of PDT treatment.

\section{ACKNOWLEDGMENTS}

This work has been carried out partially under the project TEC2006-06548/TCM of the Spanish Ministry of Education and Science.

\section{REFERENCES}

[1] Thomas H. Foster, Richard S. Murant, Robert G. Bryant, Robert S. Knox, Scott L. Gibson and Russel Hilf, "Oxygen Consumption and Diffusion Effects in Photodynamic Therapy", Radiation Research 126 (3), 296-303 (1991). 
[2] F. Fanjul-Vélez, M. del Campo-Gutiérrez, N. Ortega-Quijano and J. L. Arce-Diego, "Predictive analysis of photodynamic therapy applied to esophagus cancer”, Proceedings of SPIE vol. 6991, 699117-1, 699117-8 (2008).

[3] F. Fanjul-Vélez, O. G. Romanov, M. López-Escobar, N. Ortega-Quijano and J. L. Arce-Diego, "Necrosis prediction of Photodynamic Therapy applied to skin disorders", Proceedings of SPIE vol. 7161A, 71610P-1,71610P-8 (2009). C. Jones (private communication).

[4] Diepgen, T. L. et al., "The Epidemiology of Skin Cancer”, British Journal of Dermatology 146, 61:1-6 (2002).

[5] Xin-Hua Hu, Yuanming Feng, Jun Q. Lu, Ron R. Allison, Rosa E. Cuenca, Gordon H. Downie and Claudio H. Sibata, "Modeling of a Type II Photofrin-mediated Photodynamic Therapy Process in a Heterogeneous Tissue Phantom", Photochemistry and Photobiology 81 (6), 1460-1468 (2005).

[6] J. Mobley and T. Vo-Dinh, [Optical Properties of Tissue], CRC Press, Boca Raton, (2003).

[7] Matthias Kress, Thomas Meier, Rudolf Steiner, Frank Dolp, Rainer Erdmann, Uwe Ortmann, Angelica Rück, "Time-resolved microspectrofluorometry and fluorescente Lifetime Imaging of photosensitizers using picosecond pulsed diode lasers in laser scanning microscopes ", Journal of Biomedical Optics Vol. 8 (1), 26-32 (2003).

[8] Mark Niedre, Michael S. Petterson and Brian C. Wilson, "Direct Near-Infrared Luminescence Detection of Singlet Oxygen Generate by Photodynamic Therapy in Cells in Vitro and Tissues in Vivo", Photochemistry and Photobiology 75 (4), 382-391 (2002).

[9] Irene Georgakoudi, Michael G. Nichols and Thomas H. Foster, "The Mechanism of Photofrin Photobleaching and its Consequences for Photodynamic Dosimetry", Photochemistry and Photobiology 65 (1), 135-144 (1996).

[10] Ma, X., J. Q. Lu, H. Ding and X. H. Hu, "Bulk optical parameters of porcine skin dermis tissues at 8 wavelength from 325 to $1557 \mathrm{~nm}$ ", Opt. Lett. 30, 412-414 (2005). 